\section{An APL function for the asymptotic test of significance for Goodman and Kruskal's gamma statistic}

\section{KENNETH J. BERRY \\ Colorado State University, Fort Collins, Colorado}

Goodman and Kruskal's (1954) gamma is a measure of association that is often used to measure the degree of association between two cross-classified ordinal variables. Gamma is a symmetric measure of ordinal association that utilizes information only from concordant and discordant (i.e., untied) object pairs; Somers' (1962) $d$, on the other hand, is an asymmetric measure of ordinal association that utilizes the information from the concordant and discordant object pairs, but adjusts for additional object pairs that are tied on the dependent variable but not on the independent variable. Although these two measures examine somewhat different kinds of object pairs obtained from the two ordinal variables, they both estimate the degree to which knowledge of order on one variable predicts order on the other variable.

Several APL routines exist to compute Goodman and Kruskal's (1954) symmetric measure of ordinal association, gamma, as well as Somers' (1962) asymmetric measures, $d_{r c}$, with row variable dependent, and $d_{c r}$, with column variable dependent (Berry \& Mielke, 1986; Brennan, Nitz, \& Daly, 1988). Unfortunately, none of these routines provides the probability value, under the null hypothesis, for any of the three measures. The function GAMMA is a noniterative APL function that calculates the three coefficients of ordinal association-gamma, $d_{r c}$, and $d_{\mathrm{cr}}$-the associated two-sided probability values, and the $95 \%$ and $99 \%$ confidence limits, under full multinomial sampling.

The probability values and the confidence limits are computed using the asymptotic standard errors developed by Goodman and Kruskal $(1963,1972)$ and, specifically, do not use the approximate standard errors given in many textbooks. The approximate tests are based on the maximum standard error (Goodman \& Kruskal, 1963), which yields a conservative test with a probability of falsely rejecting the null hypothesis known only to be less than some nominal value, or they assume uniform marginals (Somers, 1980), which may yield inaccurate test results for cross classifications with severely skewed marginal distributions. Moreover, because the approximations introduced by Somers (1980) are based on null populations, they preclude the computation of confidence limits.

Procedure. Consider a full multinomial sample of $n$ discrete objects, which are cross classified by two ordi-

Correspondence may be addressed to Kenneth $\mathbf{J}$. Berry, Department of Sociology, Colorado State University, Fort Collins, CO 80523. nal variables, $r$ and $c$, with $r$ representing the row variable, $c$ representing the column variable, and $n_{i j}$ indicating the observed frequency of objects in row $i$ and column $j, 1 \leq i \leq R$, and $1 \leq j \leq C$. The cell proportions are given by $p_{i j}=n_{i j} / n$, and the row and column marginal proportions are given by

$$
p_{i} .=\sum_{j} p_{i j}
$$

and

$$
p_{\cdot j}=\sum_{i} p_{i j}
$$

respectively. Then $G$, the sample estimate of gamma, is defined as

$$
G=\frac{\Pi_{s}-\Pi_{d}}{\Pi_{s}+\Pi_{d}},
$$

with the estimated asymptotic variance of $G$ given by

$\operatorname{VAR}(G)=\frac{16}{n\left(\Pi_{s}+\Pi_{d}\right)^{4}} \sum_{i} \sum_{j} p_{i j}\left[\Pi_{s} D_{i j}-\Pi_{d} S_{i j}\right]^{2}$

where

$$
\begin{aligned}
& S_{i j}=\mathrm{I}_{i j}+\mathrm{III}_{i j}, \\
& D_{i j}=\mathrm{II}_{i j}+\mathrm{IV}_{i j}, \\
& \Pi_{s}=2 \sum_{i} \sum_{j} p_{i j} \mathrm{II}_{i j}, \\
& \Pi_{d}=2 \sum_{i} \sum_{j} p_{i j} \mathrm{IV}_{i j},
\end{aligned}
$$

and where

$$
\begin{aligned}
& \mathrm{I}_{i j}=\left\{\begin{array}{l}
0, \text { if } i=R \text { and } / \text { or } j=C \\
\sum_{a=i+1}^{R} \sum_{b=j+1}^{C} p_{a b}, \text { otherwise, }
\end{array}\right. \\
& \mathrm{II}_{i j}=\left\{\begin{array}{l}
0, \text { if } i=1 \text { and/or } j=C \\
\sum_{a=1}^{i-1} \sum_{b=j+1}^{C} p_{a b}, \text { otherwise, }
\end{array}\right. \\
& \text { III }_{i j}=\left\{\begin{array}{l}
0, \text { if } i=1 \text { and } / \text { or } j=1 \\
\sum_{a=1}^{i-1} \sum_{b=1}^{j-1} p_{a b}, \text { otherwise, }
\end{array}\right.
\end{aligned}
$$




$$
\mathrm{IV}_{i j}=\left\{\begin{array}{l}
0, \text { if } i=R \text { and } / \text { or } j=1 \\
\sum_{a=i+1}^{R} \sum_{b=1}^{j-1} p_{a b}, \text { otherwise. }
\end{array}\right.
$$

Somers's $d_{r c}$ is defined as

$$
d_{r c}=\frac{\Pi_{s}-\Pi_{d}}{1-\sum_{j} p_{{ }_{j}}^{2}}
$$

with the estimated asymptotic variance of $d_{r c}$ given by

$$
\begin{gathered}
\operatorname{VAR}\left(d_{r c}\right)=\frac{4}{n\left(1-\sum_{j} p_{\cdot j}^{2}\right)^{4}} \sum_{i} \sum_{j} p_{i j} \\
\cdot\left[\left(\Pi_{s}-\Pi_{d}\right)\left(1-p_{\cdot j}\right)-\left(1-\sum_{j} p_{\cdot j}^{2}\right)\left(S_{i j}-D_{i j}\right)\right]^{2},
\end{gathered}
$$

and Somers's $d_{c r}$ is defined as

$$
d_{c r}=\frac{\Pi_{s}-\Pi_{d}}{1-\sum_{i} p_{i}^{2}},
$$

with the estimated asymptotic variance of $d_{c r}$ given by

$$
\begin{aligned}
\operatorname{VAR}\left(d_{c r}\right) & =\frac{4}{n\left(1-\sum_{i} p_{i}^{2} \cdot\right)^{4}} \sum_{i} \sum_{j} p_{i j} \\
\cdot & {\left[\left(\Pi_{s}-\Pi_{d}\right)\left(1-p_{i}\right)-\left(1-\sum_{i} p_{i}^{2}\right)\left(S_{i j}-D_{i j}\right)\right]^{2} . }
\end{aligned}
$$

The distributions of $z_{G}=G /[\operatorname{VAR}(G)]^{1 / 2}, z_{d r c}=d_{r c} l$ $\left[\operatorname{VAR}\left(d_{r c}\right)\right]^{1 / 2}$, and $z_{d_{c r}}=d_{\mathrm{cr}} /\left[\operatorname{VAR}\left(d_{c r}\right)\right]^{1 / 2}$ are shown by Goodman and Kruskal (1972) to be asymptotically normal, when the null hypothesis is true.

Function. APL function GAMMA computes Goodman and Kruskal's $G$, Somers' $d_{r c}$, and Somers' $d_{c r}$, the associated two-sided probability values, and the $95 \%$ and 99\% confidence limits for each measure. Function GAMMA calls function ZTAB (Evans \& Gilfillan, 1986) to calculate the three probability values from the com- puted $z$ scores. The user supplies, as a right argument to function GAMMA, the observed cell frequencies of a cross classification arranged as an $R \times C$ matrix. Because the three measures are designed for ordinal variables, properly signed coefficients will be returned if the matrix is arranged with the high-high values in the upper-left cell and the low-low values in the lower-right cell of the matrix. Two examples are provided in the Appendix; the data in the first example are from Kendall and Stuart $(1967$, p. 564), and the data in the second example are from Agresti and Finlay (1986, p. 221).

Language. Function GAMMA is written in APL*PLUS/PC, Version 7.0, from STSC, Inc. IBM APL does not accept the diamond separator; enter the expressions separated by the diamonds in order from left to right. The output of function GAMMA was tested and verified against the results from the four empirical data structures referenced in Somers (1980).

Availability. A listing of function GAMMA is available from Kenneth J. Berry, Department of Sociology, Colorado State University, Fort Collins, Colorado 80523.

\section{REFERENCES}

Agrestr, A., \& Finlay, B. (1986). Statistical methods for the social sciences (2nd ed.). San Francisco: Dellen.

Berry, K. J., \& Mielke, P. W., Jr. (1986). An APL function for computing measures of association for nominal-by-ordinal and ordinalby-ordinal cross classifications. Behavior Research Methods, Instruments, \& Computers, 18, 399-402.

Brennan, J. M., Nitz, L. H., \& Daly, J. E. (1988). An APL program for measures of association for ordinal variables. Behavior Research Methods, Instruments, \& Computers, 20, 429-431.

Evans, S., \& Gilfillan, L. (1986). APL approximations for common statistical tables. Behavior Research Methods, Instruments, \& Computers, 18, 337-338.

GoOdman, L. A., \& KruSkaL, W. H. (1954). Measures of association for cross classifications. Journal of the American Statistical Association, 49, 732-764.

Goodman, L. A., \& Kruskal, W. H. (1963). Measures of association for cross classifications: III. Approximate sampling theory. Journal of the American Statistical Association, 58, 310-364.

Goodman, L. A., \& Kruskal, W. H. (1972). Measures of association for cross classifications: IV. Simplification of asymptotic variances. Joumal of the American Statistical Association, 67, 415-421.

KENDALL, M. G., \& STUART, A. (1967). The advanced theory of statistics (2nd ed., Vol. 2). New York: Hafner.

SoMERS, R. H. (1962). A new asymmetric measure of association for ordinal variables. American Sociological Review, 27, 799-811.

SOMERS, R. H. (1980). Simple approximations to null sampling variances. Sociological Methods \& Research, 9, 115-126.

\section{APPENDIX
Functions Used in the Calculation of Probability Values}

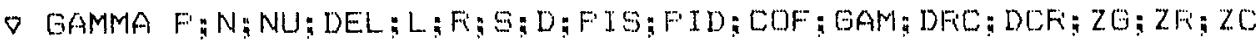

[1.] A FUNCTION GAMMA COMFUTES THE COEFFICIENTS AND ASSOCIATED

[2] A FFOBABILITY VALLES FOF GODDMAN AND FFLISKALL. $S$ GAMMA,

[.3] म SUMEF' D(RO), AND SOMERS' D(CF) TEST STATISTICS

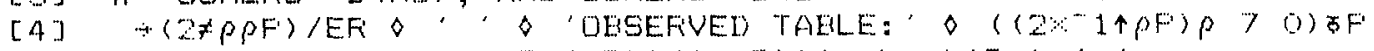

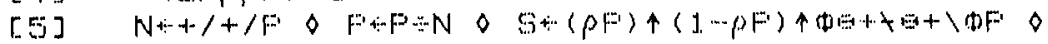

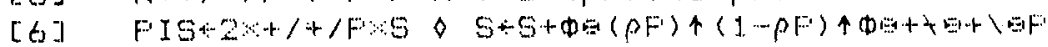

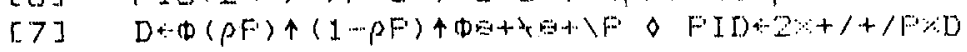




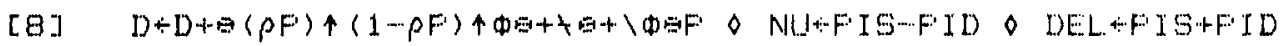

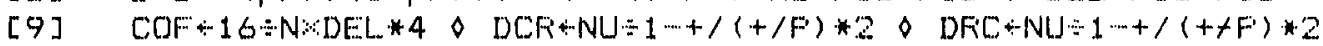

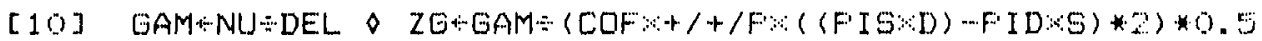

[1.1] DEL $* 1-+/(+/ F) * 2 \otimes$ COOF $-4 \div$ NODEL $* 4$

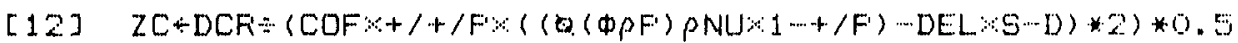

$[13] \quad D E I+1-+/(+\not P) * 2 \diamond \quad$ COF $+4 \div N \cong D E L * 4$

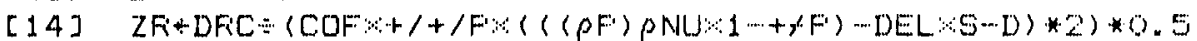

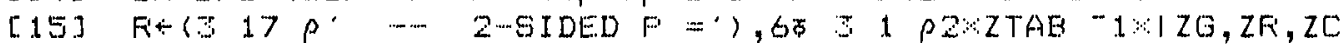

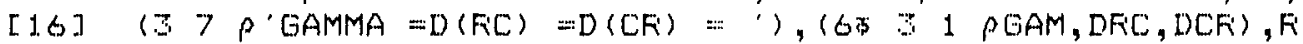

$[17] F+-1.96-2.5760 .61(G A M, D F C, D C F) \div Z G, Z F, Z C$

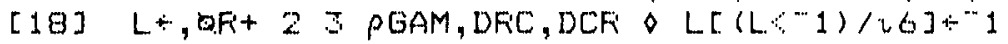

$[19]$

[20]

[2.1]

[2]

$F \div, 0(" 1 \times F)+23 \rho G A M, D F C, D C F \diamond F i(F, 1) / 1,6]+1 \diamond$

F.' COAMMA $\&(F(C) \& D(C F I)$

P- $612 \rho,(612 \rho F)$, 6 $12 \rho F \circ D+2=0.95 ?=0.99^{\circ}$

(6 $\left.2 \rho^{\prime} F^{\circ}\right)$, (6o $\left.61 \rho L\right), F,\left(6 \sigma\right.$ o $\left.1 \rho F^{\prime}\right), 610 \rho D 0+0$

[.2Z] EF: 'ERFOF: FIGHT' ARGUMENT SHOULD BE TWD DIMENSIONAL. AFIFAY'

$\nabla$

$\nabla F \div Z T A B \quad Z ; T$

[1] A FUNCTION ZTAB FETUFINS THE NOFMAL FFIOBABILITY OF VALUES $\leq Z$

[.2] F AND IS FFIOM S. EVANS AND L. GILFILLAN, EEHAVIOF FESEAFICH

[S] A METHODS, INSTFUMENTS, \& COMFUTEFS 18 (1986): 37.38.

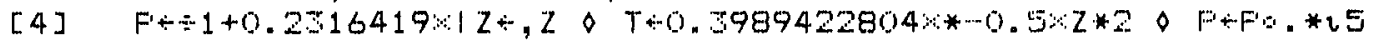

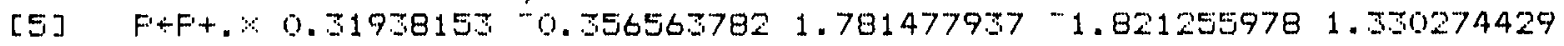

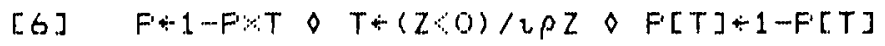

$\nabla$

EXAMFLE 1 :

(1) Enter the cedd frequencoses as a vector.

(2) Fieshape the vector to an Fi by $c$ mation.

(3) Call and execute AFL. function GAMMA.

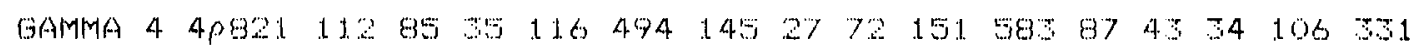

OBSERVED TABLE:

$\begin{array}{rrrr}82 . & 112 & 83 & 35 \\ 116 & 494 & 145 & 27 \\ 72 & 151 & 583 & 87 \\ 4 \% & 34 & 106 & 31\end{array}$

\begin{tabular}{|c|c|c|c|c|c|c|c|}
\hline & & .775704 & $-m$ & $2-8$ C DEE & $F$ & $\therefore$ & .0 \\
\hline & $\ldots$ & & $\ldots . . . .$. & G I IJED & & $\because=:$ & 00000 \\
\hline & & .641831 & $\cdots-\ldots . .$. & $2 \ldots-5$ ] DED & $F$ & $\because:=$ & .000000 \\
\hline
\end{tabular}

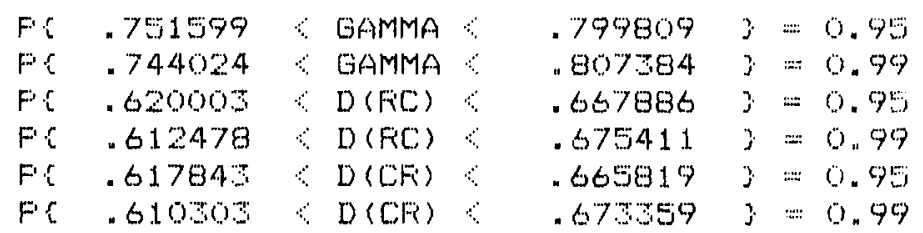

EXAMFLE 2:

(1) Enter the cell frequencies as a vector.

(2) Fieshape the vector to an $R$ by $C$ matrix.

(3) Call and execute AFL function GAMMA. 


$\begin{array}{ccc}\text { DHSEFIVED } & \text { TABLE: } & \\ 65 & 14 & 54 \\ 14 & 2 & 6 \\ 20 & 3 & 3\end{array}$

\begin{tabular}{|c|c|c|c|c|c|c|c|}
\hline GAMMA & $=\because:$ & -.481 .45 & .......... & $2 \ldots$ S IDED & $F$ & $=$ & $.00004 \mathrm{~J}$ \\
\hline$D(F C)$ & $=$ & $=.21115$ & $-\cdots$ & 2-SIDED & $F$ & $=$ & .000103 \\
\hline$D(C F i)$ & $=$ & -.26027 & $\cdots$ & $2-9$ IDED & $F$ & $=$ & .000048 \\
\hline
\end{tabular}

\begin{tabular}{|c|c|c|c|c|c|}
\hline$P r$ & $\cdots .711189$ & GAMMA & - 251501 & 3 & 0.95 \\
\hline$F:$ & -.783426 & GAMMA & $\cdots .179264$ & 3 & 0.99 \\
\hline$F=5$ & -.30661 & $D(F i C)$ & -.105565 & 3 & 0.95 \\
\hline$F !$ & . . 54462 & $D(F C)$ & $\cdots .071764$ & 3 & 0.99 \\
\hline$t ?$ & -772812 & $(D(C F)$ & .137241 & 3 & 0.95 \\
\hline 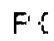 & .452976 & S D (CF) & -.097000 & $\because=$ & 0.99 \\
\hline
\end{tabular}

